\title{
On Pretopological Vector Spaces
}

\section{Madhu Ram and Shallu Sharma}

Department of Mathematics, University of Jammu, JK-180006, India; e-mail: madhuram0502@gmail.com

\begin{abstract}
The purpose of the present paper is to introduce the new class of topological spaces, namely pretopological vector spaces. We study some of the basic properties of pretopological vector spaces and investigate their relationships with certain existing spaces. Along with other results, it is proved that translation of an open (resp. closed) set in a pretopological vector space is pre-open (resp. pre-closed), that translations $(x \mapsto a+x)$ and dilations $(x \mapsto \lambda x)$ on pretopological vector spaces are precontinuous.
\end{abstract}

\section{Introduction}

The display between algebraic and topological structures was first introduced and studied by Kolmogroff in 1934 when he introduced the notion of topological vector spaces. Afterward, many researchers and mathematicians have been given innovations galore on topological vector spaces. Because of nice properties, topological vector spaces are now a prominent and fundamental notion in operator theory, fixed point theory, variational inequalities, vector equilibrium problems, etc. The researchers not only make use of topological vector spaces in other fields to develop new concepts but also stretch and extend this notion every possible way to make this field of study a more convenient and understandable. Recently, Khan et al. [8] defined s-topological vector spaces as a generalization of topological vector spaces. Khan and Iqbal [9], in 2016, put forth the concept of irresolute topological vector spaces which is independent of topological vector spaces but is included in s-topological vector spaces. Ibrahim [5] Received: March 5, 2019; Accepted: April 2, 2019 2010 Mathematics Subject Classification: 57N17, 57N99.

Keywords and phrases: pre-open sets, pre-closed sets, pretopological vector spaces.

Copyright (C) 2019 Madhu Ram and Shallu Sharma. This is an open access article distributed under the Creative Commons Attribution License, which permits unrestricted use, distribution, and reproduction in any medium, provided the original work is properly cited. 
initiated the study of $\alpha$-topological vector spaces. In 2018, the authors [18] introduced and studied another class of spaces, namely almost pretopological vector spaces. In [16, 17], we introduced the $\beta$-topological vector spaces and almost s-topological vector spaces as generalizations of s-topological vector spaces. In this paper, we introduce and study the pretopological vector spaces. Some examples of pretopological vector spaces are given. The relationship of pretopological vector spaces with certain other types of spaces are investigated.

\section{Preliminaries}

Throughout the present paper, $X$ means a topological space unless stated explicitly. For a subset $S$ of $X, C l(S)$ and $\operatorname{Int}(S)$ denote the closure and the interior of $S$ respectively. By $f: X \rightarrow Y$, we denote a mapping $f$ from a topological space $X$ to a topological space $Y$. As usual, we represent the set of real numbers by $\mathbb{R}$ and the set of complex numbers by $\mathbb{C}$. The notations $\varepsilon$ and $\delta$ denote negligibly small positive real numbers.

In 1982, the authors [12] introduced the notion of pre-open sets in topological spaces. They defined a subset $S$ of a topological space $X$ is said to be pre-open if $S \subseteq \operatorname{Int}(C l(S))$. The complement of a pre-open set in $X$ is called pre-closed; or equivalently, a subset $S$ of $X$ is pre-closed [3] if $C l(\operatorname{Int}(S)) \subseteq S$. The family of all preopen (resp. pre-closed) sets of $X$ is denoted by $P O(X)$ (resp. $P C(X)$ ). A point $x \in X$ is called pre-interior point of a subset $S$ of $X$ [6] if there exists a pre-open set $U$ in $X$ such that $x \in U \subseteq S$. The set of all pre-interior points of $S$, denoted by $\operatorname{pInt}(S)$, is called the pre-interior of $S$. In other words, the pre-interior of $S$ is the largest pre-open subset of $S$. It is known that a subset $S$ of $X$ is pre-open if and only if $S=p \operatorname{Int}(S)$. The intersection all pre-closed sets in $X$ containing $S$ is called the pre-closure of $S[3]$ and is denoted by $p C l(S)$. In other words, the pre-closure of a subset $S$ of $X$ is the smallest pre-closed set in $X$ containing $S$. It is proved in [6] that a subset $S$ of $X$ is pre-closed if and only if $S=p C l(S)$. Also, it is known that a point $x \in p C l(S)$ if and only if $S \cap U \neq \varnothing$, for every pre-open set $U$ containing $x$. It is easy to check that $p C l(S)=S \cup C l(\operatorname{Int}(S))$. A subset $S$ of $X$ is called pre-neighborhood of a point $x \in X$ if there exists a pre-open set $U$ in $X$ containing $x$ such that $U \subseteq S$. If $A \in P O(X)$ and $B \in P O(Y)$, then $A \times B \in P O(X \times Y)$, where $X$ and $Y$ are topological spaces. 
Using the concept of pre-open sets, Mashhour et al. [12] introduced a generalized form of continuous mappings, known as precontinuous mappings. They defined a mapping $f: X \rightarrow Y$ is called precontinuous if for every open set $V$ in $Y, f^{-1}(V)$ is pre-open set in $X$. Equivalently, $f$ is precontinuous if for each $x \in X$ and each open set $V$ in $Y$ containing $f(x)$, there exists a pre-open set $U$ in $X$ containing $x$ such that $f(U) \subseteq V$.

Recall that a point $x \in X$ is called a pre-interior point of $A \subseteq X$ if there exists a pre-open set $U$ in $X$ such that $x \in U \subseteq A$.

Definition 2.1. A subset $A$ of $X$ is said to be

(1) $\beta$-open [1] if $A \subseteq C l(\operatorname{Int}(C l(A)))$.

(2) semi-open [11] if $A \subseteq C l(\operatorname{Int}(A))$.

The following implications always hold but none of these implications is reversible:

$$
\begin{aligned}
& \text { open set } \rightarrow \text { semi-open set } \\
& \text { pre-open set } \rightarrow \beta \text {-open sets }
\end{aligned}
$$

For example, let $X=\mathbb{R}$ with standard topology. Consider $A=[0,1)$ and $B=$ $(0,1) \cap \mathbb{Q}$, where $\mathbb{Q}$ denotes the set of rational numbers, and $C=[0,1) \cup \mathbb{Q}$. Then $A$ is semi-open which is not open, $C$ is $\beta$-open which is not pre-open and $B$ is pre-open which is not open. Also, observe that semi-openness and pre-openness are independent notions.

Definition 2.2. Let $L$ be a vector space over the field $F$ ( $\mathbb{R}$ or $\mathbb{C}$ ). Let $\tau$ be a topology on $L$ such that:

(1) For each $x, y \in L$ and each open neighborhood $W$ of $x+y$ in $L$, there exist open neighborhoods $U$ and $V$ of $x$ and $y$ respectively, in $L$ such that $U+V \subseteq W$ and

(2) For each $\lambda \in F, x \in L$ and each open neighborhood $W$ of $\lambda x$ in $L$, there exist open neighborhoods $U$ of $\lambda$ in $F$ and $V$ of $x$ in $L$ such that $U . V \subseteq W$.

Then the pair $\left(L_{(F)}, \tau\right)$ is called topological vector space.

Definition 2.3. [8] Let $L$ be a vector space over the field $F(\mathbb{R}$ or $\mathbb{C}$ ) and let $\tau$ be a 
topology on $L$ such that:

(1) For each $x, y \in L$ and each open set $W$ in $L$ containing $x+y$, there exist semiopen sets $U$ and $V$ in $L$ containing $x$ and $y$ respectively such that $U+V \subseteq W$ and

(2) For each $\lambda \in F, x \in L$ and each open set $W$ in $L$ containing $\lambda x$, there exist semiopen sets $U$ in $F$ containing $\lambda$ and $V$ in $L$ containing $x$ such that $U . V \subseteq W$.

Then the pair $\left(L_{(F)}, \tau\right)$ is called s-topological vector space.

Definition 2.4. [9] Let $L$ be a vector space over the field $F$ ( $\mathbb{R}$ or $\mathbb{C}$ ) and $\tau$ be a topology on $L$ such that:

(1) For each $x, y \in L$ and each semi-open set $W$ in $L$ containing $x+y$, there exist semi-open sets $U$ and $V$ in $L$ containing $x$ and $y$ respectively such that $U+V \subseteq W$ and

(2) For each $\lambda \in F, x \in L$ and each semi-open set $W$ in $L$ containing $\lambda x$, there exist semi-open sets $U$ in $F$ containing $\lambda$ and $V$ in $L$ containing $x$ such that $U . V \subseteq W$.

Then the pair $\left(L_{(F)}, \tau\right)$ is called irresolute topological vector space.

Definition 2.5. [17] Let $L$ be a vector space over the field $F(\mathbb{R}$ or $\mathbb{C}$ with standard topology) and let $\tau$ be a topology on $L$ such that:

(1) For each $x, y \in L$ and each open set $W$ in $L$ containing $x+y$, there exist $\beta$-open sets $U$ and $V$ in $L$ containing $x$ and $y$ respectively, such that $U+V \subseteq W$ and

(2) For each $x \in L, \lambda \in F$ and each open set $W$ in $L$ containing $\lambda x$, there exist $\beta$-open sets $U$ in $F$ containing $\lambda$ and $V$ in $L$ containing $x$ such that $U . V \subseteq W$.

Then the pair $\left(L_{(F)}, \tau\right)$ is called $\beta$-topological vector space.

\section{Pretopological Vector Spaces}

In this section, we introduce the concept of pretopological vector spaces and present some examples of them. We briefly mention relations of pretopological vector spaces with some existing spaces. Later, we investigate some basic properties of pretopological vector spaces. 
Definition 3.1. Let $L$ be a vector space over the field $F$, where $F=\mathbb{R}$ or $\mathbb{C}$ with standard topology. Let $\tau$ be a topology on $L$ such that the following conditions are satisfied:

(1) For each $x, y \in L$ and each open set $W$ in $L$ containing $x+y$, there exist preopen sets $U$ and $V$ in $L$ containing $x$ and $y$ respectively such that $U+V \subseteq W$ and

(2) For each $\lambda \in F, x \in L$ and each open set $W$ in $L$ containing $\lambda x$, there exist preopen sets $U$ in $F$ containing $\lambda$ and $V$ in $L$ containing $x$ such that $U . V \subseteq W$.

Then the pair $\left(L_{(F)}, \tau\right)$ is called a pretopological vector space (written in short, PTVS).

\section{Examples of pretopological vector spaces}

Example 1. Let $L=\mathbb{R}$ be the vector space of real numbers over the field $F=\mathbb{R}$, where $L=F=\mathbb{R}$ is endowed with standard topology. Then $\left(L_{(F)}, \tau\right)$ is a pretopological vector space.

Obviously, every topological vector space is a pretopological vector space and every pretopological vector space is a $\beta$-topological vector space but the converses are not always true. The following is an example of pretopological vector spaces which is not a topological vector space:

Example 2. Consider the field $F=\mathbb{R}$ with standard topology. Let $L=\mathbb{R}$ be endowed with the topology $\tau=\{\varnothing, D, \mathbb{R}\}$, where $D$ denotes the set of irrational numbers. We show that $\left(L_{(\mathbb{R})}, \tau\right)$ is a PTVS. For this purpose, we have to prove the following:

(1) Let $x, y \in L$. If $x+y$ is rational, then the only open set in $L$ containing $x+y$ is $\mathbb{R}$. So, there is nothing to prove.

If $x+y$ is irrational, then for open neighborhood $W=D$ of $x+y$ in $L$. We have following cases:

Case (i). If both $x$ and $y$ are irrational, we can choose pre-open sets $U=\{x\}$ and $V=\{y\}$ in $L$ such that $U+V \subseteq W$.

Case (ii). If one of $x$ or $y$ is rational, say $y$. Then, for the selection of pre-open sets 
$U=\{x\}$ and $V=\{p, y\}$ in $L$, where $p \in D$ such that $p+x \in D$, we have $U+V \subseteq W$.

This proves the first condition of the definition of pretopological vector spaces.

(2) Let $\lambda \in \mathbb{R}$ and $x \in L$. If $\lambda x$ is rational, then it is straightforward to prove. Suppose $\lambda x$ is irrational. Let $W=D$ be an open neighborhood of $\lambda x$. The following cases arise:

Case (i). If both $\lambda$ and $x$ are irrational, then, choose pre-open sets $U=[(\lambda-\varepsilon, \lambda+\varepsilon) \cap \mathbb{Q}] \cup\{\lambda\}$ in $\mathbb{R}$ and $V=\{x\}$ in $L$, we have $U . V \subseteq W$.

Case (ii). If $\lambda$ is rational and $x$ is irrational, then for the selection of pre-open sets $U=(\lambda-\varepsilon, \lambda+\varepsilon) \cap \mathbb{Q}$ in $\mathbb{R}$ and $V=\{x\}$ in $L$, we have $U . V \subseteq W$.

Case (iii). Finally, suppose $\lambda$ is irrational and $x$ is rational. Then, for the choice of pre-open sets $U=(\lambda-\varepsilon, \lambda+\varepsilon) \cap D$ in $\mathbb{R}$ and $V=\{x, p\}$ in $L$ (where the selection of $p \in D$ is in this way that $p q$ is irrational for each $q \in U$ ), we find $U . V \subseteq W$.

Hence $\left(L_{(\mathbb{R})}, \tau\right)$ is pretopological vector space.

Remark 3.1. Definition 3.1 reflects that pretopological vector spaces are independent of irresolute topological vector spaces as well as s-topological vector spaces. For example, Example 2 is neither an irresolute topological vector space nor an s-topological vector space. Also, an example of s-topological vector spaces is stated in [8] which is not a pretopological vector space.

Henceforth, a pretopological vector space $L$ means a pretopological vector space $\left(L_{(F)}, \tau\right)$ and by a scalar, we mean an element of $F$. We discuss now some basic properties of pretopological vector spaces.

Theorem 3.1. Let $U$ be any open set in a pretopological vector space $L$. The following statements are true:

(i) $x+U \in P O(L)$, for each $x \in L$.

(ii) $\lambda U \in P O(L)$, for each scalar $\lambda \neq 0$.

Proof. (i) Let $y \in x+U$. Then $y=x+u$ for some $u \in U$. By definition of pretopological vector spaces, there exist pre-open sets $V$ and $W$ in $L$ containing $-x$ and 
$y$ respectively such that $V+W \subseteq U$. This gives $y \in W \subseteq x+U$ and hence $y \in \operatorname{pInt}(x+U) \Rightarrow x+U \subseteq \operatorname{pInt}(x+U)$. Consequently, $x+U=\operatorname{pInt}(x+U)$. That is, $x+U \in P O(L)$.

(ii) Let $x \in \lambda U$. Then $x=\lambda u$ for some $u \in U$ implies $\frac{1}{\lambda} x \in U$. By definition of pretopological vector spaces, there exist pre-open sets $W$ in the topological field $F$ and $V$ in $L$ such that $\frac{1}{\lambda} \in W, x \in V$ and $W . V \subseteq U$. Whence we find that $x \in V \subseteq \lambda U$. This shows that $x$ is pre-interior point of $\lambda U$. Since $x \in \lambda U$ was arbitrary, it follows that $\operatorname{pInt}(\lambda U)=\lambda U$. Hence $\lambda U \in P O(L)$.

Corollary 3.1.1. If $U$ is any non-empty open set and $B$ is any set in a pretopological vector space $L$, then $B+U \in P O(X)$. That is, sums of open sets in a pretopological vector space are pre-open.

Theorem 3.2. For any open set $U$ in a pretopological vector space $L$, the following hold:

(i) $x+U \subseteq \operatorname{Int}(C l(x+U))$ for each $x \in L$.

(ii) $\lambda U \subseteq \operatorname{Int}(C l(\lambda U))$ for each non-zero scalar $\lambda$.

Proof. Follows immediately from Theorem 3.1.

Theorem 3.3. Let A be any closed subset of a pretopological vector space $L$. The following statements are valid:

(i) $x+A \in P C(L)$, for each $x \in L$.

(ii) $\lambda A \in P C(L)$, for each scalar $\lambda$ with $\lambda \neq 0$.

Proof. (i) Let $y \in p C l(x+A)$. Consider $z=-x+y$ and let $W$ be an open set in $L$ containing $z$. By Definition 3.1, there exist pre-open sets $U$ and $V$ in $L$ containing $-x$ and $y$ respectively, such that $U+V \subseteq W$. Since $y \in p C l(x+A), V \cap(x+A) \neq \varnothing \Rightarrow$ there is $f \in V \cap(x+A) \Rightarrow-x+f \in(-x+x+A) \cap(U+V)=F \cap(U+V) \subseteq$ $A \cap W$ showing that $A \cap W \neq \varnothing$. Therefore, $z \in C l(A)=A$; that is $y \in x+A$. Thus, $p C l(x+A) \subseteq x+A \Rightarrow p C l(x+A)=x+A$. This proves that $x+A$ is pre-closed in $L$. That is, $x+A \in P C(L)$. 
(ii) Let $x \in p C l(\lambda A)$. Let $W$ be an open set in $L$ containing $\frac{1}{\lambda} x$. By Definition 3.1, there exist pre-open sets $U$ in $F$ containing $\frac{1}{\lambda}$ and $V$ in $L$ containing $x$ such that $U . V \subseteq W$. Since $x \in p C l(\lambda A)$, there is $f \in V \cap(\lambda A)$. This implies that $\frac{1}{\lambda} f \in$ $\left(\frac{1}{\lambda} \cdot \lambda A\right) \cap(U . V) \subseteq A \cap W \Rightarrow A \cap W \neq \varnothing \Rightarrow \frac{1}{\lambda} x \in C l(A)=A . \quad$ That $\quad$ is, $\quad x \in \lambda A$. Therefore, $p C l(\lambda A)=\lambda A$ proving that $\lambda A$ is pre-closed in $L$. Hence the assertion follows.

Theorem 3.4. For any closed set A in a pretopological vector space $L$, the following hold:

(i) $C l(\operatorname{Int}(x+A)) \subseteq x+A$ for each $x \in L$.

(ii) $C l(\operatorname{Int}(\lambda A)) \subseteq \lambda A$ for each non-zero scalar $\lambda$.

Proof. Follows immediately from Theorem 3.3.

Theorem 3.5. Let $A$ and $B$ be any subsets of a pretopological vector space $L$. Then $p C l(A)+p C l(B) \subseteq C l(A+B)$.

Proof. Let $z \in p C l(A)+p C l(B)$. Then $z=x+y$ for some $x \in \operatorname{pCl}(A)$ and $y \in p C l(B)$. Let $W$ be any open neighborhood of $z$ in $L$. By definition of pretopological vector spaces, there exist pre-open sets $U$ and $V$ in $L$ containing $x$ and $y$ respectively, such that $U+V \subseteq W$. Since $x \in p C l(A)$ and $y \in p C l(B)$, there are $f \in A \cap U$ and $g \in B \cap V \Rightarrow f+g \in(A+B) \cap(U+V) \subseteq(A+B) \cap W \Rightarrow(A+B) \cap W \neq \varnothing \quad$ and hence $z \in C l(A+B)$. Thereby the assertion follows.

Theorem 3.6. Let $A$ and $B$ be any subsets of a pretopological vector space $L$. Then $A+\operatorname{Int}(B) \subseteq \operatorname{pInt}(A+B)$.

Proof. Let $z \in A+\operatorname{Int}(B)$ be arbitrary. Then $z=x+y$ for some $x \in A$ and $y \in \operatorname{Int}(B)$. This results in $-x+z \in \operatorname{Int}(B)$ and consequently, by Definition 3.1, there exist pre-open sets $U$ and $V$ in $L$ containing $-x$ and $z$ respectively, such that $U+V \subseteq \operatorname{Int}(B) . \quad$ In $\quad$ particular, $\quad-x+V \subseteq \operatorname{Int}(B) \Rightarrow V \subseteq x+B \subseteq A+B \Rightarrow z \in$ $\operatorname{pInt}(A+B)$. Hence the proof. 
Corollary 3.6.1. Under the same assumptions of Theorem 3.6, $A+\operatorname{Int}(B) \subseteq$ $\operatorname{pInt}(A+B)$.

Presenting properties of some special functions on pretopological vector spaces.

Theorem 3.7. Let $L$ be a pretopological vector space. Then

(1) the translation mapping $J_{x}: L \rightarrow L$ defined by $J_{x}(y)=x+y, \forall y \in L$, is precontinuous.

(2) the multiplication mapping $M_{\lambda}: L \rightarrow L$ defined by $M_{\lambda}(x)=\lambda x, \quad \forall x \in L$ $(\lambda \neq 0$ is a fixed scalar $)$, is precontinuous.

Proof. (1) Let $y \in L$ be an arbitrary. Let $W$ be an open neighborhood of $x+y$ in $L$. Then there exist $U, V \in P O(L)$ such that $x \in U, y \in V$ satisfying $U+V \subseteq W$. This results in $x+V \subseteq W \Rightarrow J_{x}(V) \subseteq W$. Since $V$ is pre-open set in $L$ containing $y$, it follows that $J_{x}$ is precontinuous at $y$ and hence $J_{x}$ is precontinuous.

(2) Let $x \in L$ and $W$ be any open neighborhood of $M_{\lambda}(x)$. By definition of pretopological vector spaces, there exist pre-open sets $U$ in the topological field $F$ containing $\lambda$ and $V$ in $L$ containing $x$ such that $U . V \subseteq W$. This gives $\lambda V \subseteq W \Rightarrow M_{\lambda}(V) \subseteq W \Rightarrow M_{\lambda}$ is precontinuous at $x$. Since $x \in L$ was arbitrary, it follows that $M_{\lambda}$ is precontinuous.

Theorem 3.8. For a pretopological vector space $L$, the mapping $\phi: L \times L \rightarrow L$ defined by $\phi(x, y)=x+y$, for all $x, y \in L$, is precontinuous.

Proof. Let $x, y \in L$ and let $W$ be any open neighborhood of $x+y$. Then there exist pre-open sets $U$ and $V$ in $L$ such that $x \in U, y \in V$ and $U+V \subseteq W$. This implies that $\phi(U \times V)=U+V \subseteq W$. Since $U \times V$ is pre-open in $L \times L$ (with respect to product topology), it follows that $\phi$ is precontinuous at $(x, y)$. Since $x, y \in L$ were arbitrary, $\phi$ is precontinuous.

Theorem 3.9. For a pretopological vector space $L$, the mapping $\psi: F \times L \rightarrow L$ defined by $\psi(\lambda, x)=\lambda x$, for all $\lambda \in F, x \in L$, is precontinuous.

Proof. Let $\lambda \in F, x \in L$ be arbitrary. In order to show that $\psi$ is precontinuous, it 
suffices to show that $\psi$ is precontinuous at $(\lambda, x)$. Let $W$ be any open neighborhood of $\psi(\lambda, x)=\lambda x$. By definition of pretopological vector spaces, there exist pre-open sets $U$ in the topological field $F$ and $V$ in $L$ such that $\lambda \in U, x \in V$ and $U . V \subseteq W$. This means $\psi(U \times V)=U . V \subseteq W$. Since $U \times V$ is pre-open in $F \times L$, it follows that $\psi$ is precontinuous at $(\lambda, x)$. This completes the proof.

\section{Characterizations}

In this section, we offer some useful characterizations of pretopological vector spaces.

Theorem 4.1. For any subset A of a pretopological vector space L, the following assertions are true:

(i) $p C l(x+A) \subseteq x+C l(A)$ for each $x \in L$.

(ii) $x+p C l(A) \subseteq C l(x+A)$ for each $x \in L$.

(iii) $x+\operatorname{Int}(A) \subseteq \operatorname{pInt}(x+A)$ for each $x \in L$.

(iv) $\operatorname{Int}(x+A) \subseteq x+\operatorname{pInt}(A)$ for each $x \in L$.

Proof. (i) Let $y \in p C l(x+A)$ and consider $z=-x+y$ in $L$. Let $W$ be an open set in $L$ containing $z$. Since $L$ is pretopological vector space, there exist pre-open sets $U$ and $V$ in $L$ containing $-x$ and $y$ respectively, such that $U+V \subseteq W$. Since $y \in p C l(x+A)$, there is $g \in(x+A) \cap V$. Consequently, $-x+g \in(-x+x+A) \cap(U+V)=A \cap$ $(U+V) \subseteq A \cap W$ showing that $A \cap W \neq \varnothing$ and hence $z \in C l(A)$. That is, $y \in x+C l(A)$. Therefore, $p C l(x+A) \subseteq x+C l(A)$.

(ii) Consider $z \in x+p C l(A)$. Then $z=x+y$ for some $y \in p C l(A)$. Let $W$ be an open neighborhood of $x+y$ in $L$. Since $L$ is pretopological vector space, there exist preopen sets $U$ and $V$ in $L$ containing $x$ and $y$ respectively, such that $U+V \subseteq W$. Since $y \in p C l(A), A \cap V \neq \varnothing$. So, there is $g \in A \cap V$ and thereby $x+g \in(x+A) \cap$ $(U+V) \subseteq(x+A) \cap W$. Therefore, $(x+A) \cap W \neq \varnothing$. This implies $z \in C l(x+A)$. This proves the assertion. 
(iii) Let $y \in x+\operatorname{Int}(A)$. Then $y=x+u$ for some $u \in \operatorname{Int}(A)$ and hence there exist pre-open sets $U$ and $V$ in $L$ containing $-x$ and $y$ respectively, such that $U+V \subseteq \operatorname{Int}(A)$. Now, $-x+V \subseteq U+V \subseteq \operatorname{Int}(A) \subseteq A$ implies that $V \subseteq x+A$. Since $V$ is pre-open in $L$ containing $y$, we must have $y \in \operatorname{pInt}(x+A)$. Thus, $x+\operatorname{Int}(A) \subseteq \operatorname{pInt}(x+A)$.

(iv) Let $z \in \operatorname{Int}(x+A)$. Then $z=x+y$ for some $y \in A$. Since $L$ is pretopological vector space, there exist pre-open sets $U$ and $V$ in $L$ containing $x$ and $y$ respectively, such that $U+V \subseteq \operatorname{Int}(x+A)$. Now, $x+V \subseteq U+V \subseteq \operatorname{Int}(x+A) \subseteq x+A$ implies that $x+V \subseteq x+p \operatorname{Int}(A)$. Since $z=x+y \in x+V$, we have $z \in x+\operatorname{pInt}(A)$. Hence $\operatorname{Int}(x+A) \subseteq x+\operatorname{pInt}(A)$.

Theorem 4.2. Let $A$ be a subset of a pretopological vector space $L$. Then $p C l(x+C l(A)) \subseteq x+C l(A)$ for each $x \in L$.

Proof. Assume $y \in p C l(x+C l(A))$. Consider $z=-x+y$ and let $W$ be an open neighborhood of $z$ in $L$. Then there exist pre-open neighborhoods $U$ and $V$ of $-x$ and $y$ respectively, in $L$ such that $U+V \subseteq W$. This implies that $V \subseteq x+W$. Since $y \in p C l(x+C l(A))$, we have $V \cap(x+C l(A)) \neq \varnothing \Rightarrow(x+W) \cap(x+C l(A)) \neq \varnothing \Rightarrow$ $W \cap C l(A) \neq \varnothing$. Since $W$ is open, $W \cap A \neq \varnothing$. Hence $z \in C l(A)$, that is $y \in x+C l(A)$, Consequently, the assertion follows.

If we compare Theorem 4.2 with part (i) of Theorem 4.1, we find that the former is an improvement of the later.

Furthermore, the following result is an improvement of part (iii) of Theorem 4:1.

Theorem 4.3. For any subset $A$ of a pretopological vector space $L$, $x+\operatorname{Int}(A) \subseteq \operatorname{pInt}(x+\operatorname{pInt}(A))$ for each $x \in L$.

Proof. Let $y \in x+\operatorname{Int}(A)$. Then $y=x+u$ for some $u \in \operatorname{Int}(A)$. As a result of this, we get pre-open sets $U$ and $V$ in $L$ containing $-x$ and $y$ respectively, such that $U+V \subseteq \operatorname{Int}(A)$. Now, $-x+V \subseteq U+V \subseteq \operatorname{Int}(A) \subseteq \operatorname{pInt}(A)$ implies that $V \subseteq$ $x+\operatorname{pInt}(A)$. Since $V$ is pre-open in $L$ such that $y \in V$, we have that $y \in \operatorname{pInt}(x+\operatorname{pInt}(A))$ proving that $x+\operatorname{Int}(A) \subseteq \operatorname{pInt}(x+\operatorname{pInt}(A))$. This completes the proof. 
The analog of Theorem 4.1 is the following:

Theorem 4.4. Let $A$ and $L$ be as in Theorem 4.1. Then

(i) $p \operatorname{Cl}(\lambda A) \subseteq \lambda C l(A)$ for each non-zero scalar $\lambda$.

(ii) $\lambda p C l(A) \subseteq C l(\lambda A)$ for each non-zero scalar $\lambda$.

(iii) $\lambda \operatorname{Int}(A) \subseteq \operatorname{pInt}(\lambda A)$ for each non-zero scalar $\lambda$.

(iv) $\operatorname{Int}(\lambda A) \subseteq \lambda \operatorname{PInt}(A)$ for each non-zero scalar $\lambda$.

Definition 4.1. Let $B$ be a subset of $X$. A collection $\mathcal{U}=\left\{U_{i}: i \in I\right\}$ of pre-open sets of $X$ is called a pre-open cover of $B$ if $B \subseteq \bigcup\left\{U_{i}: i \in I\right\}$. A topological space $X$ is said to be strongly compact [13] if every pre-open cover of $X$ has a finite subcover.

A subset $B$ of $X$ is said to be strongly compact relative to $X$ if every cover of $K$ by pre-open sets of $X$ has a finite subcover.

In consequence of Theorem 3.1, we obtain the following results:

Theorem 4.5. Let $K$ be any strongly compact set in a pretopological vector space $L$. Then $x+K$ is compact, for each $x \in L$.

Proof. Let $\left\{U_{i}: i \in I\right\}$ be any open cover of $x+K$. Then $x+K \subseteq$ $\bigcup\left\{U_{i}: i \in I\right\} \Rightarrow K \subseteq \bigcup\left\{-x+U_{i}: i \in I\right\}$. By Theorem 3.1, $-x+U_{i}$ is pre-open in $L$. Consequently, $\left\{-x+U_{i}: i \in I\right\}$ is pre-open cover of $K$. Since $K$ is strongly compact, there exists a finite subset $I_{0}$ of $I$ such that $K \subseteq \bigcup\left\{-x+U_{i}: i \in I_{0}\right\}$. This implies that $x+K \subseteq \bigcup\left\{U_{i}: i \in I_{0}\right\}$. This proves that $x+K$ is compact.

Theorem 4.6. Let $L$ be a pretopological vector space. Then scalar multiple of any strongly compact subset of $L$ is compact.

Proof. Suppose that $K$ is a strongly compact set in $L$. Let $\lambda$ be any scalar. If $\lambda=0$, we are done. Assume $\lambda \neq 0$. We have to show that $\lambda K$ is compact. Let $\left\{U_{j}: j \in I\right\}$ be any open cover of $\lambda K$. Then $\lambda K \subseteq \bigcup\left\{U_{j}: j \in I\right\} \Rightarrow K \subseteq \frac{1}{\lambda} . \bigcup\left\{U_{j}: j \in I\right\}$ $\Rightarrow K \subseteq \cup\left\{\frac{1}{\lambda} U_{j}: j \in I\right\}$. Since $U_{j}$ is open in $L$, by Theorem $3.1, \frac{1}{\lambda} U_{j}$ is pre-open 
set in $L$. Consequently, $\left\{\frac{1}{\lambda} U_{j}: j \in I\right\}$ is pre-open cover of $K$. But $K$ is strongly compact, there exists a finite subset $I_{0}$ of $I$ such that $K \subseteq \bigcup\left\{\frac{1}{\lambda} U_{j}: j \in I_{0}\right\}$. It gives $\lambda K \subseteq \bigcup\left\{U_{j}: j \in I_{0}\right\}$. This proves that $\lambda K$ is compact.

\section{References}

[1] M. E. Abd El-Monsef, S. N. El-Deeb and R. A. Mahmoud, $\beta$-open sets and $\beta$-continuous mapping, Bull. Fac. Sci. Assiut. Univ. 12 (1983), 77-90.

[2] S. G. Crossley and S. K. Hildebrand, Semi-closure, Texas J. Sci. 22 (1971), 99-112.

[3] N. El-Deeb, I. A. Hasanein, A. S. Mashhour and T. Noiri, On p-regular spaces, Bull. Math. Soc. Sci. Math. R. S. Roumanie (N.S.) 27(75) (1983), 311-315.

[4] M. Ganster, Some remarks on strongly compact spaces and semi-compact spaces, Bull. Malaysian Math. Soc. 10(2) (1987), 67-81.

[5] H. Z. Ibrahim, $\alpha$-topological vector spaces, Science Journal of University of Zakho 5(1) (2017), 107-111.

[6] Y. B. Jun, S. W. Jeong, H. J. Lee and J. W. Lee, Applications of pre-open sets, Appl. Gen. Topol. 9(2) (2008), 213-228.

[7] J. L. Kelly, General Topology, New York: Van Nastrand, 1955.

[8] M. D. Khan, S. Azam and M. S. Bosan, s-topological vector spaces, J. Linear Topol. Algebra 4(2) (2015), 153-158.

[9] M. Khan and M. I. Iqbal, On irresolute topological vector spaces, Adv. Pure Math. 6 (2016), 105-112.

[10] Kolmogroff, Zur Normierbarkeit eines topologischen linearen Raumes, Studia Mathematica 5 (1934), 29-33.

[11] N. Levine, Semi-open sets and semi-continuity in topological spaces, Amer. Math. Monthly 70 (1963), 36-41.

[12] A. S. Mashhour, M. E. Abd El-Monsef and S. N. El-Deeb, On precontinuous and weak precontinuous mappings, Proc. Math. Phys. Soc. Egypt 53 (1982), 47-53.

[13] A. S. Mashhour, M. E. Abd El-Monsef, I. A. Hasanein and T. Noiri, Strongly compact spaces, Delta J. Sci. 8(1) (1984), 30-46. 
[14] A. S. Mashhour, I. A. Hasanein and S. N. El-Deep, A note on semi-continuity and precontinuity, Indian J. Pure Appl. Math. 13(10) (1982), 1119-1123.

[15] L. Narici and E. Beckenstein, Topological Vector Spaces, CRC Press, 2010.

[16] M. Ram, S. Sharma, S. Billawria and A. Hussain, On almost s-topological vector spaces, J. Adv. Stud. Topol. 9(2) (2018), 139-146.

[17] S. Sharma and M. Ram, On $\beta$-topological vector spaces, J. Linear Topol. Algeb. 8(1) (2019), 63-70.

[18] S. Sharma, M. Ram and S. Billawria, On almost pretopological vector spaces, Open Access Library Journal 5 (2018), 1-10. 